\title{
Expression of MUC2 epithelial mucin in breast carcinoma
}

\author{
M D Walsh, M A McGuckin, P L Devine, B G Hohn, R G Wright
}

\begin{abstract}
Aims-To examine the expression of the MUC2 epithelial mucin in breast carcinoma; to relate this to patient survival. Methods-Sections from 210 breast carcinomas were stained with the antiMUC2 core protein monoclonal antibody, 4F1, using an immunoperoxidase technique. The proportion of tumour cells positively stained and the localisation and intensity of any staining were recorded. Expression of MUC2 was compared with histological type and grade, tumour size, presence of nodal metastases, presence of oestrogen receptors, and menopausal status. The prognostic value of MUC2 expression was examined using Kaplan-Meier survival analysis. Results-MUC2 mucin was detected in $19 \%$ of cases of invasive carcinoma, in $11 \%$ of cases of carcinoma in situ, where present, but very rarely in adjacent normal breast epithelium. Presence of MUC2 was significantly associated with a shorter disease free interval ( $p<0.05$ ), although the observed difierence in duration of overall survival was not significant.
\end{abstract}

Conclusions-The MUC2 detected in breast carcinoma may be underglycosylated or staining may represent detection of the protein core before the completion of glycosylation. The virtual absence of 4F1 reactivity in normal breast epithelium suggests that, unlike the MUC1 mucin, the MUC2 mucin is not highly expressed by these cells. The mechanism by which expression of MUC2 affects the biology of breast tumours is unclear, although expression may be a refiection of general derepression of genes during tumour progression.

(F Clin Pathol 1993;46:922-925)

Knowledge of the cellular characteristics of breast tumours that influence prognosis is increasing. Important factors include the activation of oncogenes and the impaired activity of tumour suppressor gene products; the expression of steroid receptors and dependent proteins; growth factors and their receptors; proliferation rate, and the expression of various glycoproteins. ${ }^{1}$

Mucins are heavily glycosylated, high molecular weight glycoproteins produced by many human epithelial tissues. ${ }^{2}$ Five mucin genes have recently been cloned. These five mucins possess a region containing a variable number of tandem repeats (VNTR) in which a sequence, varying from eight to 23 amino acids, is repeated a large and variable number of times. ${ }^{3}$ The product of the MUC1 gene, polymorphic epithelial mucin, is an integral part of the membrane of breast epithelial cells and is highly expressed by breast carcinomas. ${ }^{4}$ The MUC2 gene codes for a protein produced and secreted by epithelial cells of tissues derived embryologically from endoderm, chiefly the gastrointestinal and respiratory tracts. ${ }^{6}$ Knowledge of the amino acid sequence of the MUC2 VNTR has permitted production of anti-MUC2 monoclonal antibodies using synthetic peptides as immunogens. ${ }^{78}$ Preliminary immunohistochemical studies with these antibodies showed the presence of MUC2 in tumours of the gastrointestinal and respiratory tracts, ovary, and breast. ${ }^{78}$ Monoclonal antibody 4F1 recognises a peptide epitope on the VNTR of MUC2. ${ }^{8}$ The current study was undertaken to describe more completely the expression of this mucin, using the $4 \mathrm{~F} 1$ antibody, in carcinoma of the breast, and to determine whether expression of this mucin was related to patient survival.

\section{Methods}

Two hundred and ten patients with carcinoma of the breast, diagnosed at the Department of Pathology of the Royal Brisbane Hospital between 1971 and 1986, were included retrospectively in this study. The cases were sequential and unselected other than being excluded if there was insufficient histological material or inadequate follow up information. Clinical information collected included: age, family history, size, side and site of lesion, details of nodal metastases, oestrogen receptor status (only available from 111 patients), and menopausal status. Disease free interval and overall survival were recorded for each patient; median disease free interval and overall survival durations for the group were 72 and 81 months, respectively.

All paraffin wax blocks containing tumour from each primary breast carcinoma were sectioned at $3 \mu \mathrm{m}$. Sections from each block were stained with haematoxylin and eosin, and classification of tumour type, including any carcinoma in situ component present, as well as tumour grading, ${ }^{910}$ was performed by one observer (RGW). Serial sections were 


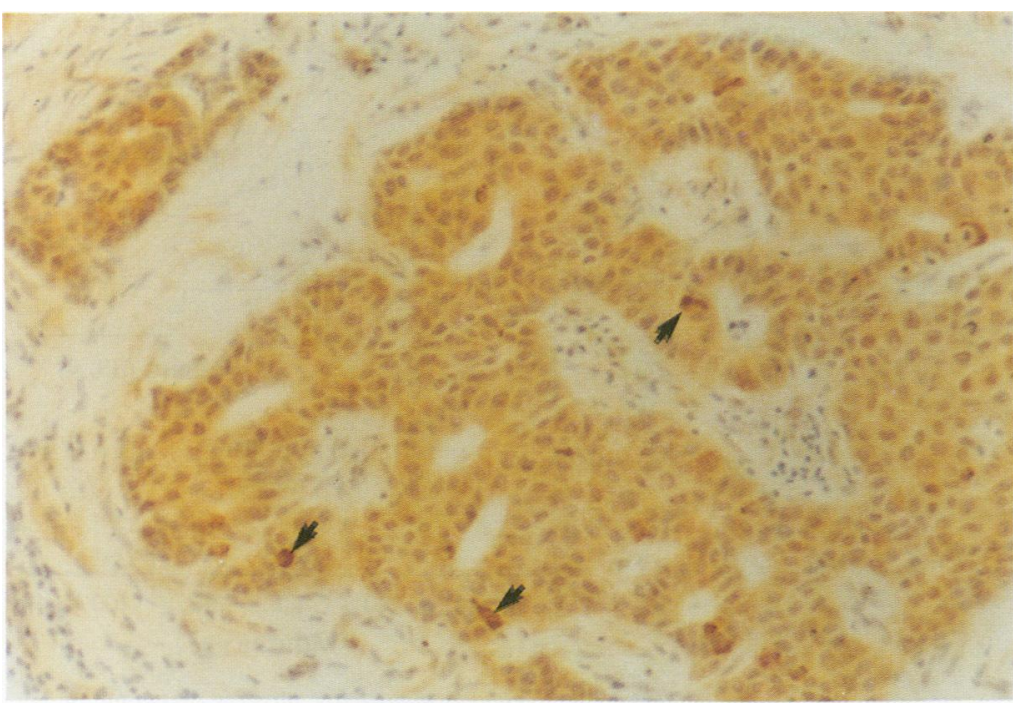

Figure 1 Grade 1 (well differentiated) infiltrating ductal carcinoma showing typical diffuse cytoplasmic staining with the $4 F 1$ antibody. Note stronger staining in isolated cells (arrows).

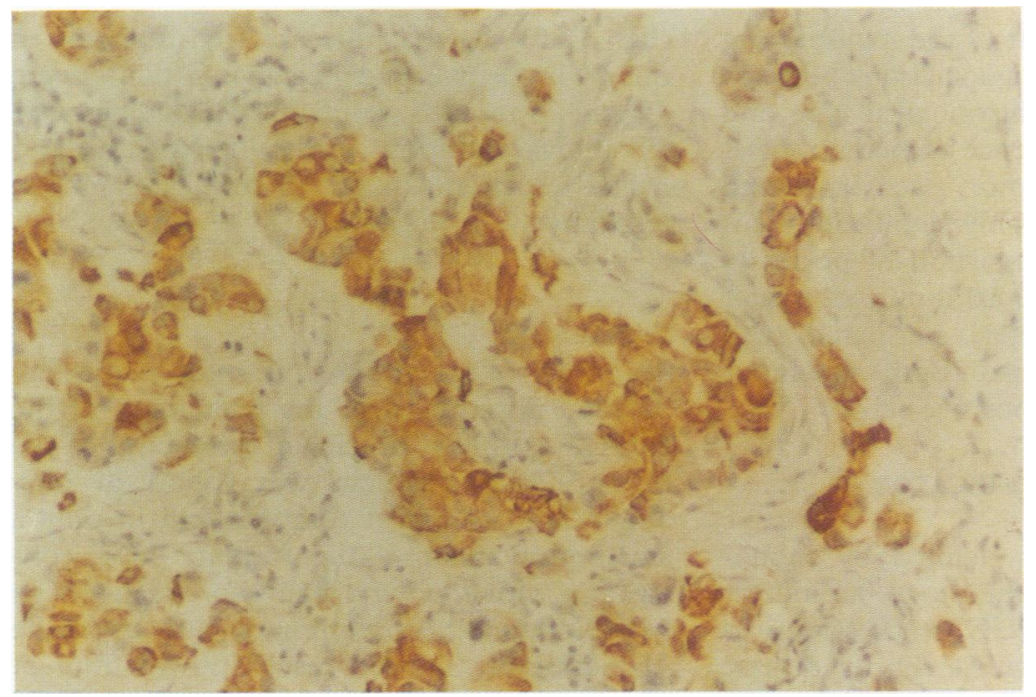

Figure 2 Poorly differentiated (grade 3) infiltrating ductal carcinoma demonstrating intense cytoplasmic reactivity.
Tumours containing $4 \mathrm{~F} 1$ positive cells were classified according to the percentage of cells positive $(1=1-25 \% ; 2=26-50 \% ; 3=$ $51-75 \% ; 4=76-100 \%$ ), the localisation of the antigen in the cell (apical membrane, membrane, cytoplasmic), and the intensity of the reaction (on a scale from 1 to 4 ) by two observers (MDW and MAM).

Clinical and histological data were recorded on a devoted database (Paradox, Borland International, Scotts Valley, California, USA). Associations between 4F1 positivity and other variables were tested using $\chi^{2}$ analysis. The correlations between $4 \mathrm{~F} 1$ positivity and disease free interval and overall survival duration were examined using Kaplan-Meier actuarial survival analysis, and tested for significant differences using the log rank test.

\section{Results}

MUC2 EXPRESSION IN NORMAL BREAST

MUC2 expression, as defined by $4 \mathrm{~F} 1$ reactivity, was not detected in normal breast epithelium, except in one case where there was weak cytoplasmic staining.

\section{MUC2 EXPRESSION IN COEXISTING CARCINOMA} IN SITU

The series showed coexisting carcinoma in situ in 81 of the 210 (39\%) invasive carcinomas; these included 74 cases of ductal carcinoma in situ and seven cases of lobular carcinoma in situ. Cytoplasmic MUC2 expression was found in $11(14 \%)$ of these cases; positive cases included 10 cases of ductal carcinoma in situ and one case of lobular carcinoma in situ. The adjacent invasive carcinoma also expressed MUC2 in seven of these cases, leaving four cases of MUC2 expression in the absence of expression in adjacent invasive cancer. Diffuse cytoplasmic staining was characteristic with no examples of membrane or extracellular MUC2 in these lesions.

stained with the $4 \mathrm{~F} 1$ monoclonal antibody using an immunoperoxidase technique. Sections were dewaxed and hydrated, treated for 10 minutes with $3 \%(\mathrm{v} / \mathrm{v}) \mathrm{H}_{2} \mathrm{O}_{2}$, in $18 \%$ (v/v) methanol, in $0.1 \mathrm{M}$ phosphate buffer, containing $0.9 \%(\mathrm{w} / \mathrm{v}) \mathrm{NaCl}(\mathrm{pH} \mathrm{7.4})$ (PBS), to limit endogenous peroxidase activity. Sections were washed three times in PBS and non-specific binding blocked in $4 \%$ skimmed milk powder in PBS for 15 minutes. After brief washing, sections were placed flat in a humidified box and coated with $4 \mathrm{~F} 1$ monoclonal antibody mouse ascites diluted 1 in 1000 in PBS for 1 hour, washed again, and coated with peroxidase conjugated rabbit anti-mouse IgM (Silenus Laboratories Pty Ltd, Australia) diluted 1 in 150 in PBS. After washing, peroxidase activity was detected using 3,3'-diaminobenzidine as chromagen with $\mathrm{H}_{2} \mathrm{O}_{2}$ as substrate. The sections were counterstained with haematoxylin and mounted.

\section{MUC2 EXPRESSION IN INVASIVE CARCINOMA}

The tumours in this series included 174 ductal carcinomas, 25 lobular carcinomas, four medullary carcinomas and seven carcinomas of other types. Expression of the MUC2 mucin, as defined by monoclonal antibody $4 \mathrm{~F} 1$, was detected in 39 of 210 (19\%) cases of invasive carcinoma. Expression was limited to less than $25 \%$ of tumour cells in $30(77 \%)$ of the positive cases; in only a single case was MUC2 expressed in more than than $75 \%$ of the tumour cells. Cytoplasmic staining was present in all positive cases; isolated weak membrane staining was seen in only a few cases; and extracellular staining was not observed. Cytoplasmic staining was usually present as diffuse staining, although in some cases intense granular staining was evident (figs 1-3). In some tumours a small proportion of positive tumour cells exhibited markedly stronger and more granular cyto- 


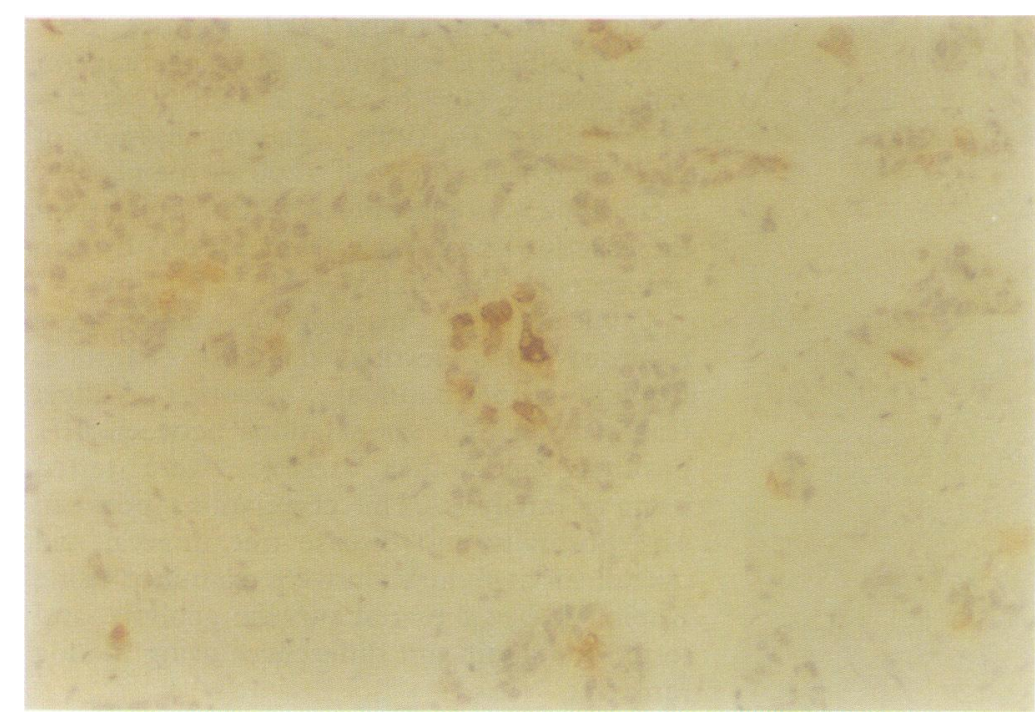

Figure 3 Grade 2 carcinoma with only occasional intensely positive cells.

plasmic staining than that of the majority (fig 1). The table details the associations between MUC2 expression and histological type and grade, tumour size, presence of nodal metastases, oestrogen receptor activity, and menopausal status. The only significant association was a higher proportion of positivity among tumours less than $10 \mathrm{~mm}$ and greater than $50 \mathrm{~mm}$ in diameter, compared with those of intermediate size. Both mucinous tumours contained 4F1-positive cells.

\section{MUC2 EXPRESSION AS AN INDICATOR OF PROGNOSIS}

Presence of MUC2, as defined by monoclonal antibody $4 \mathrm{~F} 1$, seemed to be an indicator of poor prognosis. MUC2 expression was significantly associated with a shorter disease free interval $(p<0.05)$, although the observed difference in overall survival duration was not significant $(0 \cdot 10>p>0.05)$

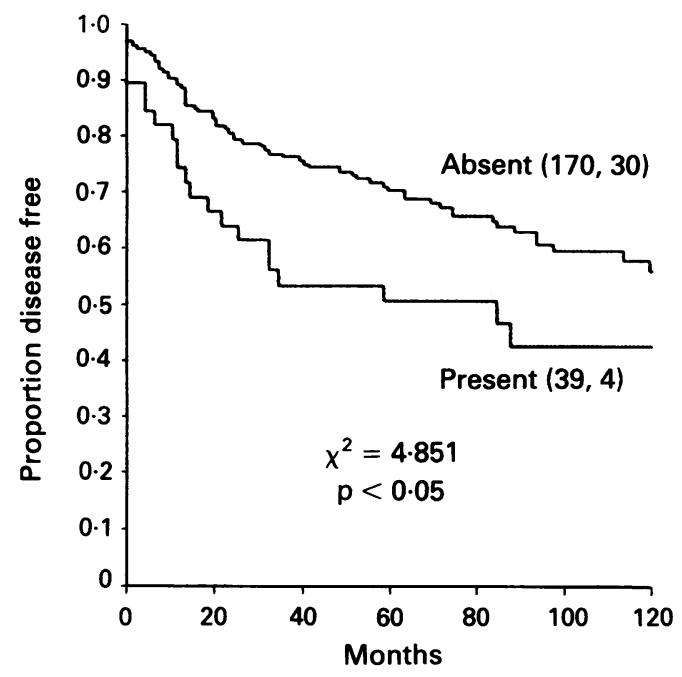

Figure 4 Ten year disease free survival curves for patients with carcinoma of the breast stratified according to the presence or absence of MUC2 expression. The number of patients in each group, followed by the number disease free after 10 years, are shown in parentheses. Log rank test, $\chi^{2}$, and $p$ value statistics are shown.

(figs 4, 5). Median disease free intervals in the $4 \mathrm{~F} 1$ positive and negative patients were 49 and 75 months, respectively. Median overall survival durations in the $4 \mathrm{~F} 1$ positive and negative patients were 73 and 82 months, respectively.

\section{Discussion}

As far as we are aware, this study is the first detailed description of the expression of the MUC2 epithelial mucin in carcinoma of the breast. MUC2 mucin was found in only about $20 \%$ of breast tumours and in only a small proportion of the tumour cells, contrasting with the very high expression of the MUC1 mucin. ${ }^{5}$ A preliminary study using the 4F1 antibody showed reactivity with four of 11 breast carcinomas, ${ }^{8}$ although MUC2 expression was not detected by immunohisto-

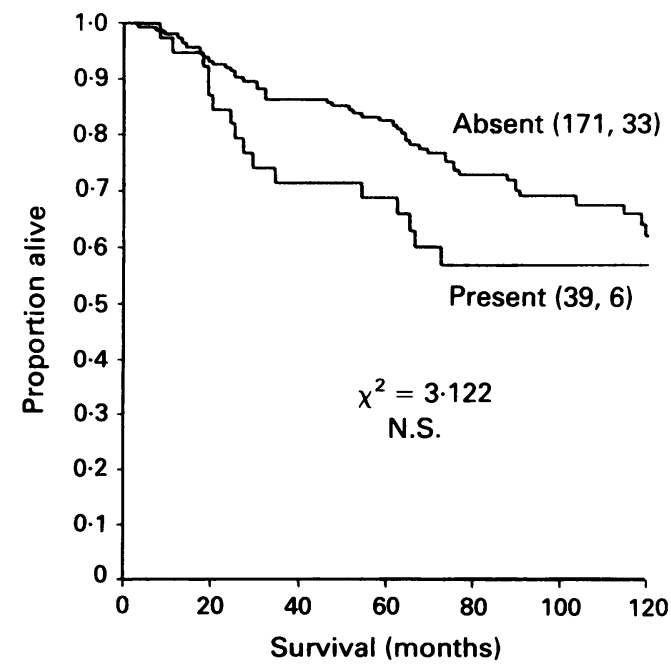

Figure 5 Ten year overall survival curves for patients with carcinoma of the breast stratified according to the presence or absence of MUC2 expression. The number of patients in each group, followed by the number alive after 10 years, are shown in parentheses. Log rank test, $\chi^{2}$, and p value statistics are shown.
Expression of MUC2, defined by antibody 4F1, related to histological type and grade, tumour size, presence of nodal metastases, oestrogen receptor activity, and menopausal status

\begin{tabular}{|c|c|c|c|c|}
\hline & \multicolumn{2}{|c|}{ Expression of MUC2 } & \multicolumn{2}{|c|}{ Statistics } \\
\hline & Absent & Present & $\chi^{2}$ & p Value \\
\hline \multicolumn{5}{|l|}{ Histology } \\
\hline Ductal & 139 & 34 & $2 \cdot 31$ & NS \\
\hline Lobular & 23 & 2 & & \\
\hline Mucinous & 0 & 2 & & \\
\hline Other & 9 & 1 & & \\
\hline \multicolumn{5}{|l|}{ Tumour grade } \\
\hline 1 & 60 & 8 & $3 \cdot 72$ & NS \\
\hline 2 & 76 & 19 & & \\
\hline 3 & 35 & 12 & & \\
\hline \multicolumn{5}{|c|}{ Tumour size (mm) } \\
\hline $1-10$ & 12 & 5 & $8 \cdot 04$ & $<0.05$ \\
\hline $11-20$ & 77 & 12 & & \\
\hline $21-50$ & 60 & 11 & & \\
\hline$>50$ & 22 & 11 & & \\
\hline \multicolumn{5}{|c|}{ Nodal metastases } \\
\hline Present & 67 & 19 & $3 \cdot 12$ & NS \\
\hline \multirow{2}{*}{\multicolumn{5}{|c|}{ Oestrogen receptor activity }} \\
\hline & & & & \\
\hline Positive & 45 & 7 & 2.49 & NS \\
\hline \multirow{2}{*}{\multicolumn{5}{|c|}{ Menopausal status }} \\
\hline & & & & \\
\hline $\begin{array}{l}\text { Pre-/peri- } \\
\text { Post- }\end{array}$ & $\begin{array}{r}24 \\
142\end{array}$ & 8 & $0 \cdot 88$ & NS \\
\hline
\end{tabular}


chemical staining in any of five breast carcinomas using three other monoclonal antibodies reactive with the VNTR region.? Polyclonal antibodies raised against deglycosylated mucin derived from colonic carcinoma were shown to react with one of five cases of breast carcinoma but not with normal breast tissue. ${ }^{11}$ These polyclonal antibodies could react with the protein core of the MUC2 mucin, although the antigenic determinants recognised have not been determined. Other evidence for expression of MUC2 includes a report of MUC2 mRNA in breast tissue. ${ }^{12}$

The examination of the prognostic value of MUC2 expression is the first such analysis in any tumour type. The mechanism by which expression of MUC2 affects the biology of breast tumours is unclear. Potential mechanisms for mucins include: a change in cellular adhesion and recognition; protection from aberrant physiological conditions such as low $\mathrm{pH}$; and interference with immune surveillance. Alternatively, expression of MUC2 may be a reflection of general loss of repression of genes during tumour progression rather than an indication of a functional role for this protein in tumour aggression.

Several studies have examined the association between expression of MUC1 mucins and survival in patients with breast cancer. ${ }^{513-18}$ The results from these studies are often contradictory, but in the largest published study increased expression of MUC1 was associated with a lower grade and better prognosis. ${ }^{5}$ The observation in the current study that MUC2 expression was present in a higher proportion of tumours of less than 10 $\mathrm{mm}$ in diameter and greater than $50 \mathrm{~mm}$ in diameter than in those of intermediate size is difficult to explain. Together with the nonsignificant trends toward a higher expression in tumours of poor grade and in tumours from patients with nodal metastases, higher expression in large tumours supports the appearance of MUC2 expression during tumour progression.

MUC2 mucin is characteristically secreted by both normal and malignant gastrointestinal cells. ${ }^{11}{ }^{19}$ The $4 \mathrm{~F} 1$ antibody reacts with an epitope that may be masked when the mucin is glycosylated. In normal colonic goblet cells 4F1 reacts strongly with the perinuclear region but not with the mucin droplet, suggesting reactivity with the protein core before the completion of glycosylation but not with the mature mucin. ${ }^{8}$ Therefore, the MUC2 detected in breast carcinoma may be underglycosylated or staining may represent detection of the protein core before glycosylation has been completed. Similarly, although extracellular MUC2 was not detected in any breast carcinomas, this may represent failure to detect highly glycosylated secreted mucin. The virtual absence of $4 \mathrm{~F} 1$ reactivity in normal breast epithelium suggests that, unlike the MUC1 mucin, the MUC2 mucin is not highly expressed by these cells. An examination of fetal and lactating breast is warranted to determine the expression during all phases in the life history of breast epithelium. MUC2 was reported as being weakly expressed in one of two cases of normal breast by immunohistochemical staining on fresh tissues using other MUC2 reactive monoclonal antibodies ${ }^{7}$; further analysis with these antibodies is also warranted.

This study has detailed the expression of the MUC2 epithelial mucin in carcinoma of the breast. Further investigations are required to elucidate the roles, if any, of this mucin in normal breast epithelial function and in the malignant characteristics of tumour cells which express the protein. The demonstration of poorer survival in patients with tumours expressing this mucin is of biological interest, but this prognostic indicator seems to be too insubstantial to have a role in clinical practice.

1 Underwood JCE. Prognostic indices in epithelial neoplasms. Rec Adv Histopathol 1992;15:17-36.

2 Zotter S, Hageman PC, Lossnitzer A, Mooi WJ, Hilgers J. Tissue and tumour distribution of human polymorphic epithelial mucin. Cancer Rev 1988;11-12:55-101.

3 Devine PL, McKenzie IFC. Mucins: structure, function, and association with malignancy. Bioessays 1992;14: 619-25.

4 Gendler SJ, Lancaster CA, Taylor-Papadimitriou J, et al. Molecular cloning and expression of human tumorassociated polymorphic epithelial mucin. $\mathcal{F}$ Biol Chem associated polymorp

5 Ellis IO, Bell J, Todd JM, et al. Evaluation of immunoreactivity with monoclonal antibody NCRC11 in breast carcinoma. Br f Cancer 1987;56:295-9.

6 Gum JR, Byrd JC, Hicks JW, et al. Molecular cloning of human intestinal mucin CDNAs. Sequence analysis and evidence for genetic polymorphism. I Biol Chem 1989; 264:6480-7

7 Xing P-X, Prenzoska J, Layton GT, Devine PL, McKenzie IFC. Second-generation monoclonal antibodies to intestinal MUC2 peptide reactive with colon bodies to intestinal MUC2 pep

8 Devine PL McGuckin MA, Birrell GW, Whitehead RH, Sachdev GP, Shield P, Ward BG. Second generation monoclonal antibodies reacting with the MUC2 mucin core protein. Br f Cancer 1993;67:1182-8.

9 Elston CW, Ellis IO. Pathology and breast screening. Histopathology 1990;16:109-18.

10 Page DL, Anderson TJ. Diagnostic histopathology of the breast. Edinburgh: Churchill Livingstone, 1987.

11 Yan P-S, Ho SB, Itzkowitz SH, Byrd JC, Siddiqui B, Kim YS. Expression of native and deglycosylated colon cancer mucin antigens in normal and malignant epithelial tissues. Lab Invest 1990;62:698-706.

12 Jany BH, Gallup MW, Yan P-S, Gum JR, Kim YS, Basbaum CB. Human bronchus and intestine express the same mucin gene. $\mathcal{f}$ Clin Invest 1991;87:77-82.

13 Wilkinson MJS, Howell A, Harris M, et al. The prognostic significance of two epithelial membrane antigens expressed by human mammary carcinomas. Int $\mathcal{f}$ Cancer 1984;33:299-304.

14 Berry N, Jones DB, Smallwood J, et al. The prognostic value of the monoclonal antibodies HMFG1 and HMFG2 in breast cancer. Br $\mathcal{F}$ Cancer 1985;51:179-86.

15 Angus B, Napier J, Purvis J, et al. Survival in breast cancer related to tumour oestrogen receptor status and immunohistochemical staining for NCRC11. $₹$ Pathol immunohistochemi.

16 Ellis IO, Hinton CP, MacNay J, et al. Immunocytochemical staining of breast carcinoma with the monoclonal antibody NCRC11: a new prognostic indicator. BrMed F 1985;290:881-3.

17 Wright C, Angus B, Napier J, et al. Prognostic factors in breast cancer: immunohistochemical staining for SP1 and NCRC11 related to survival, tumour epidermal growth factor receptor and oestrogen receptor status. $\mathcal{F}$ Pathol 1987;153:325-31.

18 Walker RA. Assessment of milk fat globule membrane antibodies and lectins as markers of short-term prognosis in breast cancer. Br $\mathcal{F}$ Cancer 1990;62:462-6.

19 Devine PL, Layton GT, Clark BA, et al. Production of evine PL, Layton GT, Clark BA, et al. Production of Biochem Biophys Res Comm. 1991;178:593-9. 